\title{
Interchain tube pressure effect in extensional flows of oligomer diluted nearly monodisperse polystyrene melts
}

\author{
Rasmussen, Henrik K.; Huang, Qian
}

Published in:

Rheologica Acta

Link to article, DOI:

$10.1007 /$ s00397-014-0757-3

Publication date:

2014

Link back to DTU Orbit

Citation (APA):

Rasmussen, H. K., \& Huang, Q. (2014). Interchain tube pressure effect in extensional flows of oligomer diluted nearly monodisperse polystyrene melts. Rheologica Acta, 53(3), 199-208. https://doi.org/10.1007/s00397-014$0757-3$

\section{General rights}

Copyright and moral rights for the publications made accessible in the public portal are retained by the authors and/or other copyright owners and it is a condition of accessing publications that users recognise and abide by the legal requirements associated with these rights.

- Users may download and print one copy of any publication from the public portal for the purpose of private study or research.

- You may not further distribute the material or use it for any profit-making activity or commercial gain

- You may freely distribute the URL identifying the publication in the public portal

If you believe that this document breaches copyright please contact us providing details, and we will remove access to the work immediately and investigate your claim. 


\title{
Interchain tube pressure effect in extensional flows of oligomer diluted nearly monodisperse polystyrene melts
}

\author{
Henrik Koblitz Rasmussen ${ }^{1}$ and Qian Huang ${ }^{2}$ \\ (1) Department of Mechanical Engineering and \\ (2) Department of Chemical and Biochemical Engineering, \\ Technical University of Denmark, DK-2800 Kgs. Lyngby, Denmark
}

\begin{abstract}
We have derived a constitutive equation to explain the extensional dynamics of oligomer diluted monodisperse polymers, if the length of the diluent has at least two Kuhn steps. These polymer systems have a flow dynamics which distinguish from pure monodisperse melts and solutions thereof, if the solvent has less than two Kuhn steps, e.g. is not a chain. The constitutive equation is based on a phenomenological tube-based model within the methodology of the molecular stress function approach. The nonlinear dynamics have been explained as a consequence of a constant thermal interchain pressure originating from the short polymer chains (e.g. the oligomers) on the wall of the tube containing the long chains. The nonlinear dynamics are uniquely defined by the Rouse time and the maximal extensibility of the long polymer chains. Both are linked to the entanglement length. The relation between the Rouse times and entanglements have been established based on published extensional experiments on nearly monodisperse polystyrene melts. The constitutive equation has shown agreement with the experimental startup of and steady extension data from Huang et al. (2013) based on $285 \mathrm{~kg} / \mathrm{mole}$ and $545 \mathrm{~kg} /$ mole polystyrenes diluted in styrene oligomers containing $3.3(1.92 \mathrm{~kg} / \mathrm{mole})$ and $7.3(4.29 \mathrm{~kg} / \mathrm{mole})$ Kuhn steps.
\end{abstract}

\section{Introduction}

The shaping of molten polymers in a polymer production into plastic products is of key importance for our way of life. The understanding of the complex flow behaviour of polymer melts is needed to predict the design of polymer processing operations. This field was initiated by phenomenological based differential constitutive equations models as the Giesekus (1962) and later the Phan-Thien and Tanner (1977) [34] model. These are versatile models which still are widely used to describe the flow on entangled polymer systems. They preceded the attempt to obtain an exact understanding of the physics of the dynamics of polymers initiated by Doi and Edwards (1978) [13]. It was based on the tube idea by P. G. De Gennes (1971) [11]. More recent developments involve the introduction of stochastically based models (Park et al. 2012) [31], although these models sometime are identical to an integral approach as the Doi and Edwards equation. The idea behind these theoretical efforts is to understand the complex flow behaviour of polymer solutions and particular polymer melts.

In experimental efforts extensional measurements are of particularly interest, as they are very sensitive to changes in flow behaviour. This was particularly seen in the measurements on (nearly) monodisperse polymer melts (Bach et al. 2003b) [3] and corresponding entangled solutions (Bhattacharjee 2002) [7]. These measurements were expected to represent the same flow physics in line with the preceding theoretical developments, but the extensional viscosities of the melts differed 
profoundly from the corresponding solutions. It motivated the recent theoretical developments (Andreev et al. 2013; Yaoita et al. 2012; Park et al. 2012) $[1,46,31]$ in an effort to create an unified theoretical framework. This effort was challenged by the recent experimental work by Huang et al. (2013a; 2013b) [18, 17] measuring the extensional viscosities on styrene oligomer diluted polystyrenes. These measurements showed that entangled polymer systems have more complex flow physics than expected. Particular the increase of the oligomer length above two Kuhn steps changes the dynamics significantly. Nearly monodisperse polymer melts diluted with an oligomer with a length of two Kuhn step or larger differ from the dynamics of both solutions and pure melts (Rasmussen et al. 2013) [37]. Here we will try to quantify the dynamics of these dilutions. Although these oligomer dilutions are theoretical ideal materials they resemble most polymer melt systems closely.

\section{Rouse time}

The ideas of the confining tube of De Gennes (1971) [11] and the constitutive model by Doi and Edwards (1978) [13] are based on a purely orientational stress. The model by Marrucci and Grizzuti (1988) [24] introduced chain stretch into the tube model. It quantified a strain hardening regime for flow faster than the transition rate $1 / \tau_{R}$, where $\tau_{R}$ commonly is referred to as the Rouse time. It always needs to be evaluated relative to the maximal relaxation time $\tau_{\max }$ of the ideally monodisperse polymer, in a dilution or not. The maximal relaxation time, relative to the transition from the flow to the glassy regime, follows the well-established relation of $\tau_{\max } \propto Z^{3.4}$ (Milner and McLeish 1998) [26], where $Z$ is the number of entanglements of the polymer. $Z=M / M_{e}$, where $M_{e}$ is the entanglement molecular weight and $M$ the molecular weight of the polymer. An evaluation of the Rouse time (e.g. $\tau_{R} / \tau_{\max }$ ) depends highly on the used theoretical concept (Larson et al. 2003; Osaki et al. 2000; Osaki et al. 2001; Likhtman and McLeish 2002; Menezes and Graessley 1982) [20, 29, 30, 21, 25] whereas all definitions imply a unique dependence of the entanglement number for a particular type of polymer.

The actual values of the Rouse time depend on the used theoretical approach. Experimentally we use uniaxial extensional measurements on nearly monodisperse melts. These melts are theoretical the most ideal polymer and uniaxial extension flow is highly sensitive to strain hardening. We use the method introduced by Wagner et al. (2005; 2010) [43, 44] where the Rouse time is obtained by fitting the following model directly to the extensional measurements. This approach predicts, within the experimental accuracy, all the extensional data and not only the onset of the initiation of strain hardening, which commonly is used to identify the Rouse time in rheometry. The stress tensor, $\sigma_{\mathrm{ij}}$, is given as

$$
\sigma_{i j}=\int_{-\infty}^{t} M\left(t-t^{\prime}\right) f\left(\boldsymbol{x}, t, t^{\prime}\right)^{2} 5\left\langle\frac{\left[\mathbf{E}\left(\boldsymbol{x}, t, t^{\prime}\right) \cdot \mathbf{u}\right]\left[\mathbf{E}\left(\boldsymbol{x}, t, t^{\prime}\right) \cdot \mathbf{u}\right]}{\left|\mathbf{E}\left(\boldsymbol{x}, t, t^{\prime}\right) \cdot \mathbf{u}\right|^{2}}\right\rangle d t^{\prime},
$$

where the stretch evolution is of the interchain pressure (Marrucci and Ianniruberto 2004) [23] type

$$
\frac{\partial}{\partial t} f\left(\boldsymbol{x}, t, t^{\prime}\right)=f\left(\boldsymbol{x}, t, t^{\prime}\right) \frac{\partial}{\partial t}\left\langle\ln \left|\mathbf{E}\left(\boldsymbol{x}, t, t^{\prime}\right) \cdot \mathbf{u}\right|\right\rangle-\frac{f\left(\boldsymbol{x}, t, t^{\prime}\right)^{2}}{3 \tau_{R}}\left(f\left(\boldsymbol{x}, t, t^{\prime}\right)^{3}-1\right) .
$$

The initial value $f\left(\boldsymbol{x}, t^{\prime}, t^{\prime}\right)=1 . M\left(t-t^{\prime}\right)$ is the memory function containing the linear dynamics of the polymer. All the angular brackets are a unit sphere integral defined as $\langle\ldots\rangle=1 /(4 \pi) \int_{|\mathbf{u}|=1} \ldots d \mathbf{u}$ where $\mathbf{u}$ is a unit vector. Analytical formulas involving all the unit sphere integrals can be found in Urakawa at al. (1995) [41] for the relevant extensional flows. The components of the displacement gradient tensor $\mathbf{E}\left(\boldsymbol{x}, t, t^{\prime}\right)$ are $E_{\mathrm{ij}}\left(\boldsymbol{x}, t, t^{\prime}\right)=\partial x_{\mathrm{i}} / \partial x_{\mathrm{j}}{ }^{\prime}, i=1,2,3$ and $j=1,2,3$ in Cartesian coordinates. $\left(x_{1}^{\prime}, x_{2}^{\prime}, x_{3}^{\prime}\right)$ are the coordinates of a particle in the reference state displaced to the coordinates $\left(x_{1}, x_{2}, x_{3}\right)$ in the present state at time $t^{\prime}$ and $t$ respectively. 
This approach has been used previously by Wagner et al. (2005) [43] on narrow molecular weight distributed (NMMD) polystyrene melts. Here we will apply it on a larger number and broader range of molecular weight of NMMD polystyrene melts. Further in the determination of the linear dynamics of our samples we apply the method by Baumgaertel, Schausberg and Winter (BSW) (Baumgaertel et al. 1990) [6], actually in the version introduced by Baumgaertel and Winter (1992) [5]. The memory function is

$$
\begin{aligned}
M\left(t-t^{\prime}\right) & =\int_{0}^{\infty} \frac{H(\tau)}{\tau^{2}} e^{\left(-\left(t-t^{\prime}\right) / \tau\right)} d \tau \\
H(\tau) & =n_{e} G_{N}^{0}\left[\left(\frac{\tau}{\tau_{\max }}\right)^{n_{e}}+\left(\frac{\tau}{\tau_{c}}\right)^{-n_{g}}\right] h\left(1-\tau / \tau_{\max }\right) .
\end{aligned}
$$

$h(x)$ is the Heaviside step function, where $n_{e}, n_{g}, \tau_{c}$ and $G_{N}^{0}$ are parameters with a unique value for each type of polymer. $\tau_{c}$ is the time scale for the transition to the glassy regime and $G_{N}^{0}$ the plateau modulus.

Table 1 lists the relevant parameters for the NMMD polystyrenes at $130^{\circ} \mathrm{C}$. The origin of the measured data is quoted in the table.

Most of the needed BSW parameters can be found in Rasmussen et al. (2013) [37]. The remaining BSW parameters are obtained by data fittings to the BSW model using the method from Rasmussen et al. 2000 [36]. The measurements with these corresponding BSW fittings are shown in figure 1. As in Rasmussen et al. (2013) [37] we use the fixed values $n_{e}=0.2$ and $n_{g}=0.7$ for all involved polystyrene samples including the dilutions. Further we use the entanglement molecular weight of $M_{e}=13.3 \mathrm{~kg} /$ mole (Bach et al. 2003b) [3] for polystyrene, where $Z=M_{w} / M_{e}$ is the number of entanglements. $M_{w}$ and $M_{n}$ are the weight and number average molecular weight respectively. The Kuhn step length, $M_{K}$, defined in term of molecular weight, is $0.6 \mathrm{~kg} /$ mole according to Fang at al. (2000) [15] for polystyrene. Notice with the exception of the $51.7 \mathrm{~kg} / \mathrm{mole}$ (PS52k) and 102.8 $\mathrm{kg} /$ mole (PS103k) polystyrenes we have applied the plateau modulus of $G_{N}^{0}=250 \mathrm{kPa}$ at $130^{\circ} \mathrm{C}$ (Bach at al. 2003b) [3].

Table 1: NMMD Polystyrene melt parameters at $130^{\circ} \mathrm{C}$.

\begin{tabular}{l|rrrrrrr}
\hline Name & Reference & $M_{w}$ & $M_{w} / M_{n}$ & $M_{w} / M_{K}$ & $G_{N}^{0}$ & $\tau_{\max }$ & $\tau_{c}$ \\
\hline PS2k & Huang et al. (2013a) [18] & $1.92 \mathrm{~kg} / \mathrm{mole}$ & 1.08 & 3.3 & - & - & - \\
PS4k & Huang et al. (2013b) [17] & $4.29 \mathrm{~kg} / \mathrm{mole}$ & 1.04 & 7.3 & - & - & - \\
\hline & & & $M_{w} / M_{e}$ & & & - \\
\hline PS52k & Nielsen et al. (2006) [28] & $51.7 \mathrm{~kg} / \mathrm{mole}$ & 1.026 & 3.9 & $330 \mathrm{kPa}$ & $13 \mathrm{~s}$ & $0.25 \mathrm{~s}$ \\
PS103k & Nielsen et al. (2006) [28] & $102.8 \mathrm{~kg} / \mathrm{mole}$ & 1.022 & 7.7 & $290 \mathrm{kPa}$ & $160 \mathrm{~s}$ & $0.35 \mathrm{~s}$ \\
PS145k & Nielsen et al. (2008) [27] & $145 \mathrm{~kg} / \mathrm{mole}$ & 1.03 & 10.9 & $250 \mathrm{kPa}$ & $660 \mathrm{~s}$ & $0.6 \mathrm{~s}$ \\
PS200k & Bach et al. (2003b) [3] & $200 \mathrm{~kg} / \mathrm{mole}$ & 1.04 & 15.0 & $250 \mathrm{kPa}$ & $1900 \mathrm{~s}$ & $0.3 \mathrm{~s}$ \\
PS285k & Huang et al. (2013a) [18] & $285 \mathrm{~kg} / \mathrm{mole}$ & 1.09 & 21.4 & $250 \mathrm{kPa}$ & $9500 \mathrm{~s}$ & $0.6 \mathrm{~s}$ \\
PS390k & Bach et al. (2003b) [3] & $390 \mathrm{~kg} / \mathrm{mole}$ & 1.06 & 29.3 & $250 \mathrm{kPa}$ & $18000 \mathrm{~s}$ & $0.35 \mathrm{~s}$ \\
PS545k & Huang et al. (2013a) [18] & $545 \mathrm{~kg} / \mathrm{mole}$ & 1.12 & 41.0 & $250 \mathrm{kPa}$ & $85000 \mathrm{~s}$ & $0.6 \mathrm{~s}$
\end{tabular}

Figure 2a-f show the startup of extensional viscosities, $\bar{\eta}^{+}$, with a constant extension rate, $\dot{\epsilon}$. This extensional viscosity is defined as $\bar{\eta}^{+}=\left(\sigma_{33}-\sigma_{11}\right) / \dot{\epsilon}$ where $x_{3}$ represents the direction of the extension. The strain is here given as $\epsilon(t)=\dot{\epsilon} \cdot t$, where the extension is initiated at time $t=0$. All extension measurements presented in this paper were performed in the Filament Stretching Rheometer (FSR) 
Table 2: Parameters for oligomer diluted NMMD polystyrenes from Huang et al. (2013a; 2013b) $[18,17]$ at $130^{\circ} \mathrm{C} . \theta$ is the weight fraction of the long chain in the dilution. $T$ is the temperature of the extensional measurements. $a_{T}$ is the time-temperature superposition shift factor from $T$ to $130^{\circ} \mathrm{C}$.

\begin{tabular}{l|rrrrrr}
\hline Polystyrenes & $\theta$ & $Z$ & $\tau_{\max }$ & $\tau_{c}$ & $T$ & $a_{T}$ \\
PS545k/PS4k & 0.525 & 21.5 & $2700 \mathrm{~s}$ & $0.25 \mathrm{~s}$ & $129^{\circ} \mathrm{C}$ & 1.22 \\
PS545k/PS2k & 0.58 & 23.8 & $1200 \mathrm{~s}$ & $0.06 \mathrm{~s}$ & $120^{\circ} \mathrm{C}$ & 8 \\
PS285k/PS2k & 0.72 & 15.4 & $440 \mathrm{~s}$ & $0.09 \mathrm{~s}$ & $120^{\circ} \mathrm{C}$ & 8 \\
PS285k/PS2k & 0.44 & 9.4 & $26 \mathrm{~s}$ & $0.03 \mathrm{~s}$ & $110^{\circ} \mathrm{C}$ & 56
\end{tabular}

(Bach et al. 2003a) [4]. In this FSR a cylindrical shaped molten sample, with initial axial height $L_{\mathrm{i}}$ and radius $R_{\mathrm{i}}$, is attached to two parallel solid cylindrical rods. A controlled separation of these rods extends the sample. The strain is obtained as $\epsilon(t)=2 \ln \left(R_{0} / R(t)\right)$ where $R(t)$ and $R_{0}$ are present (at time $t$ ) and initial $(t=0)$ central radius of the sample, respectively. $R_{0}$ may be smaller than $R_{\mathrm{i}}$ as the sample may have been pre-stretched. In the calculation of the (average) extensional stress the method from Rasmussen et al. (2013) is applied. It is calculated as

$$
\sigma_{33}-\sigma_{11}=\frac{F(t)+m_{f} g / 2}{\pi R(t)^{2}} \cdot \frac{1}{1+\left(R(t) / R_{i}\right)^{14 / 3} /\left(3\left(L_{i} / R_{i}\right)^{2}\right)} .
$$

$F(t)$ is the total axial force, $g$ the gravitational acceleration and $m_{f}$ the weight of the sample e.g. the filament. This formula ensures, within $3 \%$, a correct evaluation of the initial extensional stress, as an extra shear contribution may add to the measured elongational force during the initiation of the extension in the FSR. For further discussions please refer to (Spiegelberg 1996; Szabo 1997; Kolte at al. 1997; Rasmussen et al. 2010) [39, 40, 19, 35]. We have applied equation (5) on all presented extensional measurements. This is particularly important on the PS52k, PS103k, PS145k, PS200k and PS390k data as these preceded the appearance of equation (5).

The best possible functional relation between the entanglement number and the Rouse time seems to be an equation given as

$$
\tau_{\text {max }} / \tau_{R}=20 \cdot(Z / 21.4)^{2},
$$

It is based on the start-up of extension measurements on the NMMD polystyrene melts shown in figure 2a-f. These figures also include the actual fittings using equation (1)-(4) based on this relation in equation (6). The calculated extensional viscosities, $\bar{\eta}^{+}$, with a constant extension rate, $\dot{\epsilon}$, are the solid lines on the figures. This formula (6) indicates a relation of $\tau_{R} \propto \tau_{\max } Z^{-2} \propto Z^{3.4} Z^{-2}=Z^{1.4}$ which is somewhat lower exponent that the traditional theoretical expectation of $Z^{2}$. It may be due the low number of entanglements in these NMMD melts covering the range of about 4 to 41 .

Figure 3 includes startup of extension data followed by stress relaxation. The stress relaxation is initiated a strain of $\epsilon_{0}$ where the strain value is fixed (in figure 3 at a strain of 3), e.g. zero extension rate. The viscosity during the stress relaxation is defined similar to the startup viscosity as $\bar{\eta}^{-}=$ $\left(\sigma_{33}-\sigma_{11}\right) / \dot{\epsilon}$ where $\dot{\epsilon}$ is the extension rate during the startup of the flow. These measurements are currently the only one published for stress relaxation of NMMD polystyrene. They are of particularly interest as the time derivative or rate term in equation (2) is zero during stress relaxation. Therefore the theoretical agreement with the stress relaxation data confirms both a stretch evolution of the 'interchain pressure' type and the actual value of the Rouse time. 


\section{$3 \quad$ Interchain pressure}

As discussed in Rasmussen at al. (2013) we have to distinguish not only between polymer melts and solutions, but there is also a change of flow physics if the used diluent contains two or more Kuhn steps. An oligomer containing less than two Kuhn steps is not a chain. For polystyrene the molecular weight of one Kuhn step, $M_{K}$, is $0.6 \mathrm{~kg} / \mathrm{mole}$ (Fang at al. 2000) [15] and it is expected to be independent of a dilution with an ideal solvent.

The diluent, with at least two Kuhn steps, is assumed to be in a random state as the time dynamics of the short chains are much faster than the flow. Its random motion will impose a thermal pressure on the tube wall of the larger molecule. Doi and Edwards (1986) [14] provided the following relation for the thermal pressure on the tube wall

$$
p=\frac{\pi^{2}}{3} \frac{N b^{2}}{L_{V}^{2}} \frac{k T}{V}
$$

This relation is now a classical kinetic theory. $T$ is the temperature, $k$ Boltzmann constant, where $k T$ represents the thermal energy. $N$ is the number of Kuhn segments in the short chain, where $b$ is the length of these segments. $V$ is the volume, where $L_{V}$ is its length dimension, containing a short chain.

Equation 2 was based on the assumption that in a monodisperse melt all chains are surrounded by the same type of chains affecting each other during the extension [23]. It changes the thermal pressure on the tube wall during flow. Assuming that all the surrounding chains are in a random configuration lead to another dynamic. Inspecting equation 7 the consequence is a constant thermal pressure, $p_{0}$. This constant pressure is imposed by the short diluent chains in the direction orthogonal to the tube interface. The pressure, $p_{0}$, acts on the tube wall containing the long molecule with a force

$$
F_{s}=(\pi a L) \cdot p_{0} \cdot(1-\theta)
$$

or

$$
2 \beta \cdot F_{s} /(\zeta \pi a L(1-\theta))=\beta \cdot\left(2 p_{0} / \zeta\right) .
$$

$L$ and $a$ are the length and diameter of a tube containing the long polymer, where $\theta$ is the weight fraction of the long chain in the dilution. It is expected that both the short chains and the long ones are the same type of polymer. $\zeta$ is the friction coefficient. $\beta$ is a nonzero number. This force, $F_{s}$, is assumed to be opposed by the friction of the long chain against the short ones inside the tube

$$
F_{l}=\zeta \dot{\epsilon}\left(\pi a^{2} L / 4\right) \cdot(1-\theta)
$$

or

$$
2 \beta \cdot F_{l} /(\zeta a \pi L(1-\theta))=\beta \cdot \dot{\epsilon} a / 2 .
$$

The friction is here expected to be dependent on the volume of the tube, as the long chain slides along the random oriented short ones inside the tube.

At limited deformation the tube diameter changes its radius as a combined effect of affine deformation $(\beta=1)$ and some amount of convective constraint release (Marrucci 1996) [22] as

$$
\frac{d}{d t} a=-\beta \cdot \dot{\epsilon} a / 2
$$

where $1 \geq \beta>0$. Convective constraint release is expected to eliminate obstacles with the same rate as in the extension. This reduces the effect of the affine contraction of the tube. 
The thermal pressure originating from the short polymers does not account for the rate term. It is therefore expected to be related to the friction term alone. The proportionality

$$
\frac{d}{d t} a=-\beta \cdot \dot{\epsilon} a / 2+\beta \cdot\left(2 p_{0} / \zeta\right)\left(1-a / a_{0}\right)
$$

follows, as the thermal pressure opposes the friction. The $\left(1-a / a_{0}\right)$ term ensures equilibrium at zero extension rate as $a_{0}$ is the equilibrium value of $a$.

A variety of constitutive concepts have been suggested in the past decades. Here we follow the method of Wagner et al. (2005; 2010) using the molecular stress function (MSF) approach. Its use needs a change of variables in equation (13). The inverse of the relative tube diameter is replaced with the molecular stress function $f=a_{0} / a$ and the terms connected to the extension rate, $\dot{\epsilon}$, need to be replaced with an appropriate scalar description. We then obtain

$$
\frac{d f}{d t}=f \frac{\partial}{\partial t}\langle\ln |\mathbf{E} \cdot \mathbf{u}|\rangle-\frac{2 \beta p_{0}}{a_{0} \zeta} f(f-1)
$$

or as the Rouse time represents the onset of the strain hardening

$$
\frac{d f}{d t}=f \frac{\partial}{\partial t}\langle\ln |\mathbf{E} \cdot \mathbf{u}|\rangle-\frac{f(f-1)}{\tau_{R}}
$$

where $\tau_{R}$ is the Rouse time. Notice that the dependence of $\boldsymbol{x}, t$ and $t^{\prime}$ on $f$ is omitted in the notation. This equation for diluted melts is distinctly different from the corresponding constitutive equation for polymer solutions where the friction is balanced by a linear spring force (Pearson et al. 1989; Pearson et al. 1990) $[32,33]$. In a MSF formulation this is

$$
\frac{d f}{d t}=f \frac{\partial}{\partial t}\langle\ln |\mathbf{E} \cdot \mathbf{u}|\rangle-\frac{(f-1)}{\tau_{R}} .
$$

according to Wagner et al. (2010).

\section{Maximum extensibility}

Figure 4 shows the start up of extensional viscosity for the most diluted, $\theta=0.44$, polystyrene $(44 \%$ PS285k in 56\% PS2k) sample from Huang [18]. Here $\theta$ represents the weight fraction of the long chain in the dilution. We have added the theoretical prediction of equation (2), (15) and (16). The Rouse time, $\tau_{R}$, uniquely defines the nonlinear dynamics of these equations. $\tau_{R}$ is expected, as in the linear dynamics, to depend solely on entanglement number, $Z$. For polystyrene this relation is given by equation (6). The entanglement molecular weight in the diluted polystyrenes is expected to be $M_{e, \theta}=M_{e} / \theta$ as our diluent is the most ideal one (Huang et al. 2013a) [18]. It follows that $Z=M / M_{e, \theta}=\left(M / M_{e}\right) \theta$. The Rouse time is then calculated using equation (6). The linear viscoelastic parameters are given in table 2 based on the data from Huang et al. (2013a; 2013b) $[18,17]$. With one exception these were fitted by Rasmussen et al. (2013) [37]. The remaining data fittings are shown in figure 1. Note that the plateau modulus for the diluted samples is $G_{e, \theta}^{0}=\theta^{2} G_{N}^{0}$ according to Huang et al. (2013a) [18] where $G_{N}^{0}=250 \mathrm{kPa}$ at a reference temperature of $130^{\circ} \mathrm{C}$.

The difference between the extensional dynamics of pure NMMD melts and diluted samples was experimentally documented by Huang et al. (2013a; 2013b) [18, 17]. So as expected the melt dynamics characterized by equation (2) are up to a factor of five below the extensional measurements. The well-known Rouse type of behaviour, represented by equation (16), overestimates the stress level at all rates. A tube model based on the idea of a constant interchain pressure (CIP) in equation (15) 
seems to capture the strain hardening accurately, at low and intermediate extension rates. At higher extension rates the lack of a maximum extensibility results in a severe increase in the extensional viscosity. To account for this effect we evaluate the maximal relative stretch, $\lambda_{\max }$, of a molecule given as the square root of the number of Kuhn steps between entanglements. The Kuhn step length $\left(M_{K}\right)$ defined in term of molecular weight, is $0.6 \mathrm{~kg} / \mathrm{mole}$ (Fang at al. 2000) [15] for polystyrene. As the Kuhn step length does not change with dilution $\lambda_{\max }^{2}=M_{e, \theta} / M_{K}=22 / \theta$. To handle the transition to the maximum extensibility we use a relative Padé approximation of the nonlinear spring coefficient (Cohen 1991) [9]. The relative Padé inverse Langevin function (Ye and Sridhar 2005) [47] used is $c(f)=\left(3-f^{2} / \lambda_{\max }^{2}\right)\left(1-1 / \lambda_{\max }^{2}\right) /\left(\left(3-1 / \lambda_{\max }^{2}\right)\left(1-f^{2} / \lambda_{\max }^{2}\right)\right)$. Added into equation (15) and (16) these are represented as

$$
\frac{d f}{d t}=f \frac{\partial}{\partial t}\langle\ln |\mathbf{E} \cdot \mathbf{u}|\rangle-\frac{f(c(f) f-1)}{\tau_{R}}
$$

and

$$
\frac{d f}{d t}=f \frac{\partial}{\partial t}\langle\ln |\mathbf{E} \cdot \mathbf{u}|\rangle-\frac{(c(f) f-1)}{\tau_{R}} .
$$

(Ye and Sridhar 2005) [47], respectively.

The prediction of these two models is presented in figure 5. The figure shows the start up of extensional data from figure 4 (Huang et al. 2013a) [18]. At high rates the models give similar results and are both in agreement with the measured startup of extensional viscosity. The difference is at intermediate rates where the Rouse type of model in equation (18) still overpredicts the viscosity severely, where the CIP model (equation (17)) gives a good agreement with the measurements.

The amount of diluent in a sample is important. It can be argued that a diluent concentration of $1-\theta=0.56$ is fairly large as one would expect a contribution to the stress from the interaction in between the long polymer chains, which is not present in the CIP model in equation (17). We undoubtedly would see it in the limit of vanishing concentration of diluent where the physics of a pure melt will appear. In figure 6 similar measurements as in figure 5 are shown, decreasing the concentration of the PS2k diluent to $1-\theta=0.28$. The reduction of the solvent concentration with this factor of two changes the Rouse time but seems not to change the underlying physics. In figure 6 we do see the same extensional dynamics as in the previous figure 5. The pure melt dynamics represented by equation (2) are severely below and the Rouse type of extensional dynamics in equation (18) above the data. The CIP model is capable of predicting the measured viscosities. The amount of diluent needs to be reduced even more to create a significant deviation from the CIP assumption.

Figure 7 shows the agreement of the CIP model in equation (17) with the start-up data of a $58 \%$ PS545k in 42\% PS2k measured by Huang et al. (2013a). This sample contains 23.8 entanglements which is considerably higher than the 9.4 and 15.4 in figure 5 and 6 respectively. These entanglement numbers are measured by Huang et al. (2013a). The 47.5\% PS545k in 52.5\% PS4k sample has about 21.5 entanglement (Huang et al. 2013b). This is about $10 \%$ less than the 23.8 entanglements sample in figure 7 . Theoretically the 21.5 entanglement dynamics are therefore characterized by slightly larger $\tau_{\max } / \tau_{R}$ and $\lambda_{\max }$ compared to figure 7 . These changes are too small to distinguish experimentally between the two samples. The PS4k oligomer contains $7.3 \mathrm{Kuhn}$ steps. It is a fairly long chain in term of Kuhn steps but still considerably less than the entanglement length but larger than the $3.3 \mathrm{Kuhn}$ step of the the PS2k oligomer. Comparing with the CIP model in figure 8 we obtain a good agreement with the start-up of extension data from Huang et al. (2013b) [17], indicating an independency in the length of the oligomer on the flow physics. This independency is only for an increasing number of Kuhn steps. As concluded in Rasmussen et al. (2013) [37] the Rouse type of behaviour in equation (18) seems to represent the flow physics of a dilution with an oligomer containing less than two Kuhn step. An oligomer with less than two Kuhn steps is no longer a chain and it is functioning as a solvent. 
It is of course important that the used constitutive approach represents an appropriate description of the flow physics, not only in extension but also in shear. There is according to our knowledge no large strain shear experiment available on oligomer diluted polymers. We have inserted the corresponding start-up of shear flow to the extensional experiments in figure 6 based on the CIP model (equation (17)) in figure 9. These are qualitative similar to the observed start-up of shear flow of NMMD polymer melts (Schweizer et al. 2004, Auhl et al. 2008, Boukany et al. 2009).

Finally it is important to notice that our particularly use of the MSF constitutive concept is not a unique method. Other constitutive approaches are likely to achieve similar results (Dhole et al. 2009; van Ruymbeke et al. 2010) [12, 42]. See also the work by Yaoita et al. (2011) [45].

\section{Summary and conclusion}

We have derived a constitutive equation to predict the flow dynamics of oligomer diluted monodisperse polymers, if the length of the diluent has at least two Kuhn steps. Particular the nonlinear dynamics have been explained as a consequence of a constant interchain pressure, originating from the short polymer chains, upon the tube containing the long chains. The constitutive equation has shown quantitative agreement with the extensional experiments on styrene oligomer diluted nearly monodisperse polystyrenes from Huang et al. (2013). The lengths of the diluents were at least two Kuhn steps. These polymer systems have a flow dynamics which distinguish from pure monodisperse melts and solutions thereof, if the solvent has less than two Kuhn steps, e.g. is not a chain.

Common polymer melts are polydisperse materials. They contain in most cases a fraction of polymers which are in a random state, as the time dynamics of the short chains in the melt usually are much faster than the flow. Further, these polymer melts do normally not contain solvents. This also includes oligomers with less than two Kuhn steps. Common polymer melts neither belong to the monodisperse type of melts nor are a solution. An oligomer, with a length of at least two Kuhn steps, diluted polymer melt represents an idealized polymer melt system. 


\section{$6 \quad$ Figure Captions}

Figure 1: Loss, $G^{\prime \prime}$ (open circles; o and open triangles; $\triangle$ ) and storage moduli, $G^{\prime}$ (bullets; $\bullet$ and full triangles; $\mathbf{\Delta})$, both as a function of the angular frequency $\omega$ at $130^{\circ} \mathrm{C}$. The solid lines $(-)$ are the least-square fittings to the BSW model in equation (4). The data from left to the right curves are PS390k (Bach et al. [3]), PS145k (Nielsen et al. [27]), PS103k and PS52k (Nielsen et al. 2006) [28]. The triangles are the PS285k/PS2k blend with $\theta=0.44$ from Huang et al. [18].

Figure 2: The startup of extensional measurements, $\bar{\eta}^{+}$, as a function of the strain based time at $130^{\circ} \mathrm{C}$. The solid lines (_) are the corresponding prediction to the data from the equations (2). The dotted line $(\cdots)$ is the linear viscoelastic predictions based on the parameters listed in table 1 using equation (4). (a) PS52k, (b) PS103k, (c) PS200k, (d) PS285k, (e) PS390k and (f) PS545k.

Figure 3: The startup of extensional measurements, $\bar{\eta}^{+}$followed by stress relaxation data $\bar{\eta}^{-}$, initiated a strain of $\epsilon_{0}=3$, as a function of the time. The sample is the PS145k melt measured at a temperature of $120^{\circ} \mathrm{C}$. The time-temperature superposition shift factor from $120^{\circ} \mathrm{C}$ to $130^{\circ} \mathrm{C}$ is 23 . The solid lines $(-)$ are the corresponding prediction to the data from the equations $(2)$. The dotted line $(\cdots)$ is the linear viscoelastic predictions based on the parameters listed in table 1 using equation (4).

Figure 4: The startup of extensional measurements, $\bar{\eta}^{+}$, as a function of the strain based time at $110^{\circ} \mathrm{C}$. The PS285k/PS2k with $\theta=0.44(Z=9.4)$ data are from Huang et al. 2013 [18] measured at $110^{\circ} \mathrm{C}$. The solid lines (-) are the corresponding predictions to the data from equation (15). The dashed lines (- - ) are the corresponding predictions to the data from the equations (16). The dotted-dashed lines (-.--) are the corresponding predictions to the data from the equations (2). The dotted line $(\cdots)$ is the linear viscoelastic predictions based on the parameters listed in table 2 using equation (4).

Figure 5: The startup of extensional measurements, $\bar{\eta}^{+}$, as a function of the strain based time at $110^{\circ} \mathrm{C}$. The PS285k/PS2k with $\theta=0.44(Z=9.4)$ data are from Huang et al. 2013 [18] measured at $110^{\circ} \mathrm{C}$. The solid lines $(-)$ are the corresponding predictions to the data from equation $(17)$. The dashed lines (- - ) are the corresponding predictions to the data from the equations (18). The dotted-dashed lines (-.--) are the corresponding predictions to the data from the equations (2). The dotted line $(\cdots)$ is the linear viscoelastic predictions based on the parameters listed in table 2 using equation (4).

Figure 6: The startup of extensional measurements, $\bar{\eta}^{+}$at $120^{\circ} \mathrm{C}$, as a function of the strain based time. The data are measured on the PS285k/PS2k sample with $\theta=0.72(Z=15.4)$ from Huang et al. 2013 [18]. The startup data are from Huang et al. 2013 [18]. The solid lines (-) are the corresponding predictions to the data from equation (17). The dashed lines (- - -) are the corresponding predictions to the data from the equations (18). The dotted-dashed lines (--.--) are the corresponding predictions to the data from the equations $(2)$. The dotted line $(\cdots)$ is the linear viscoelastic predictions based on the parameters listed in table 2 using equation (4). 
Figure 7: The startup of extensional measurements, $\bar{\eta}^{+}$, as a function of the strain based time and the time at $120^{\circ} \mathrm{C}$. The PS545k/PS2k with $\theta=0.58(Z=23.8)$ data are from Huang et al. 2013 [18] measured at $120^{\circ} \mathrm{C}$. The solid lines ( - ) are the corresponding predictions to the data from equation (17). The dotted line $(\cdots)$ is the linear viscoelastic predictions based on the parameters listed in table 2 using equation (4).

Figure 8: The startup of extensional measurements, $\bar{\eta}^{+}$, as a function of the strain based time and the time at $129^{\circ} \mathrm{C}$. The PS545k/PS4k with $\theta=0.525(Z=21.5)$ data are from Huang et al. 2013 [18] measured at $129^{\circ} \mathrm{C}$. The solid lines $(-)$ are the corresponding predictions to the data from equation (17). The dotted line $(\cdots)$ is the linear viscoelastic predictions based on the parameters listed in table 2 using equation (4).

Figure 9: The startup of extensional as in figure 6 where the data are measured on the PS285k/PS2k sample with $\theta=0.72(Z=15.4)$ from Huang et al. 2013 [18]. The dashed lines (- - $)$ are the startup of the shear viscosity, $\eta^{+}$, as a function of the time, $t$, at the same value of the shear rates $\dot{\gamma}$ as the extensional rates in figure 6 . The dotted lines $(\cdots)$ are the linear viscoelastic predictions based on the parameters listed in table 2 using equation (4). 


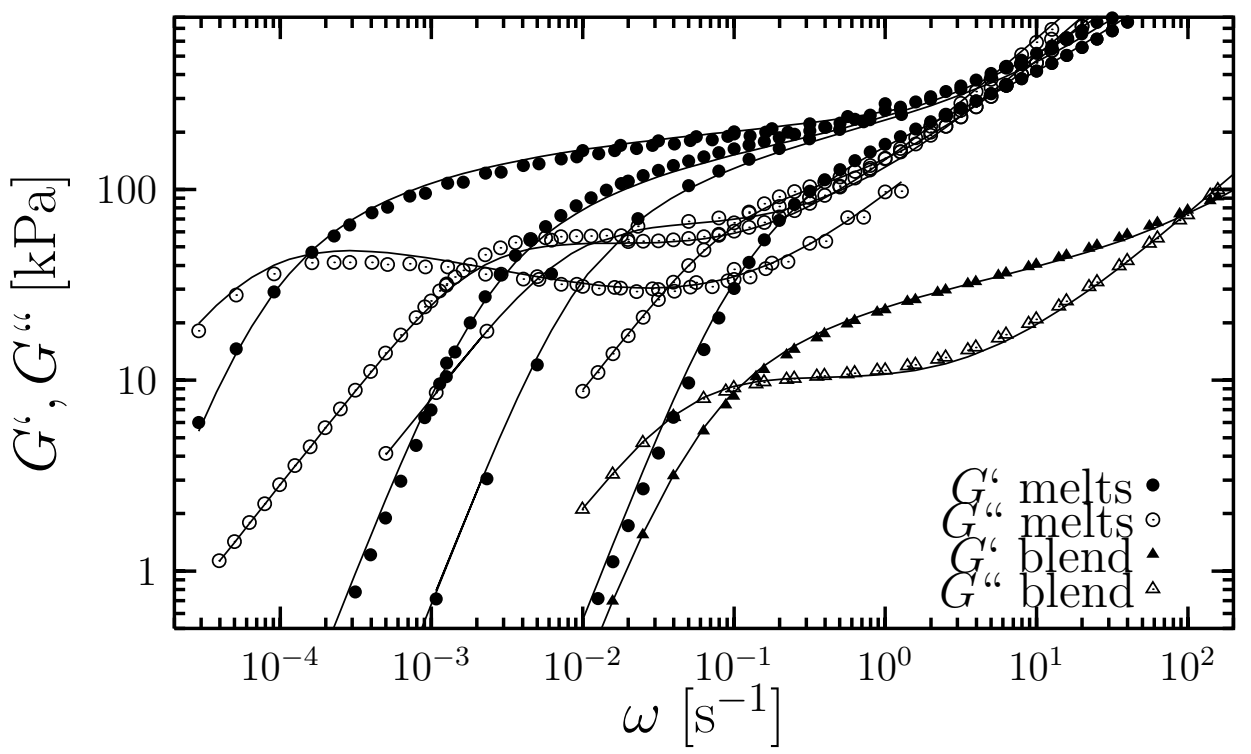

Figure 1: 


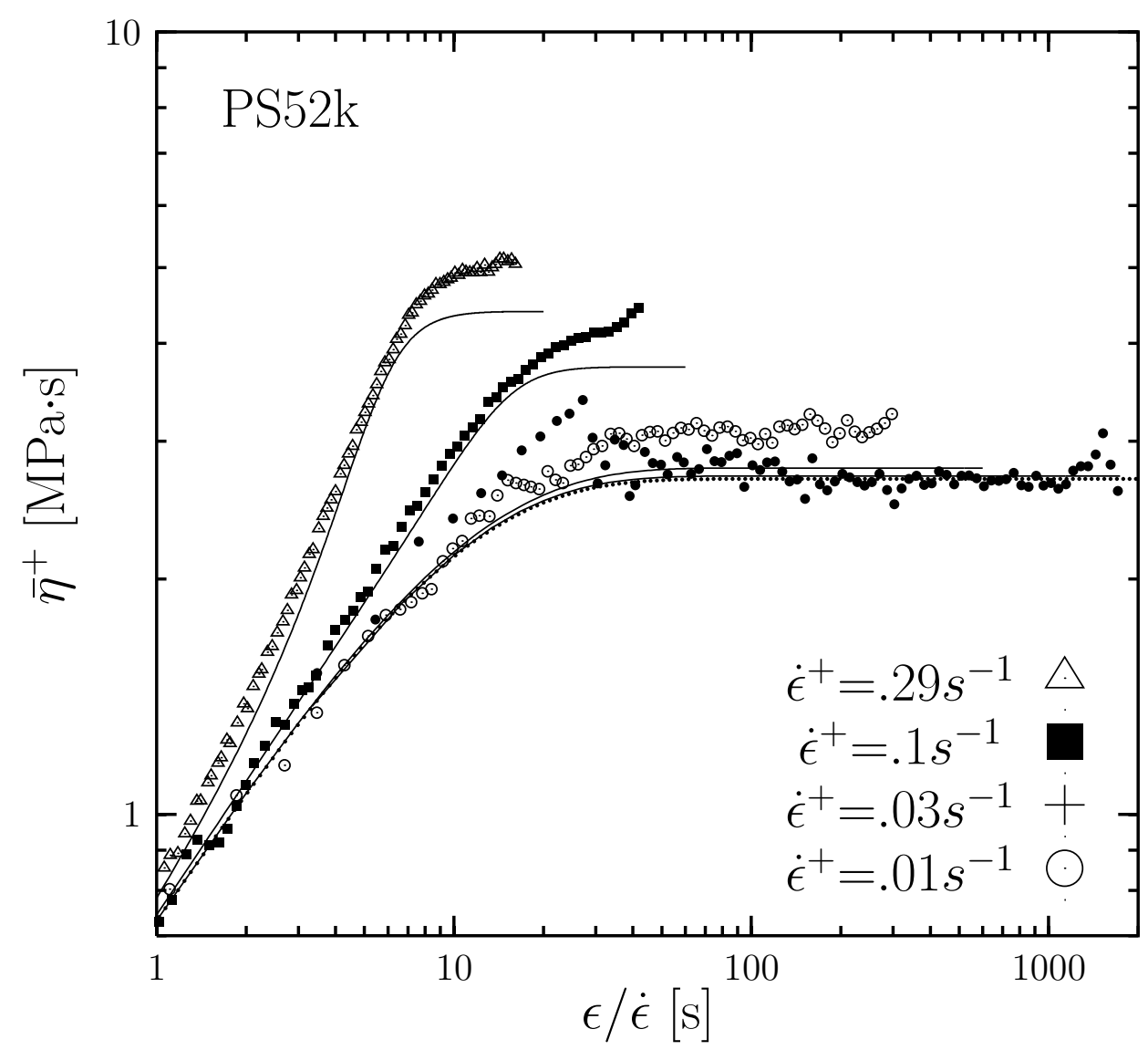

(a)

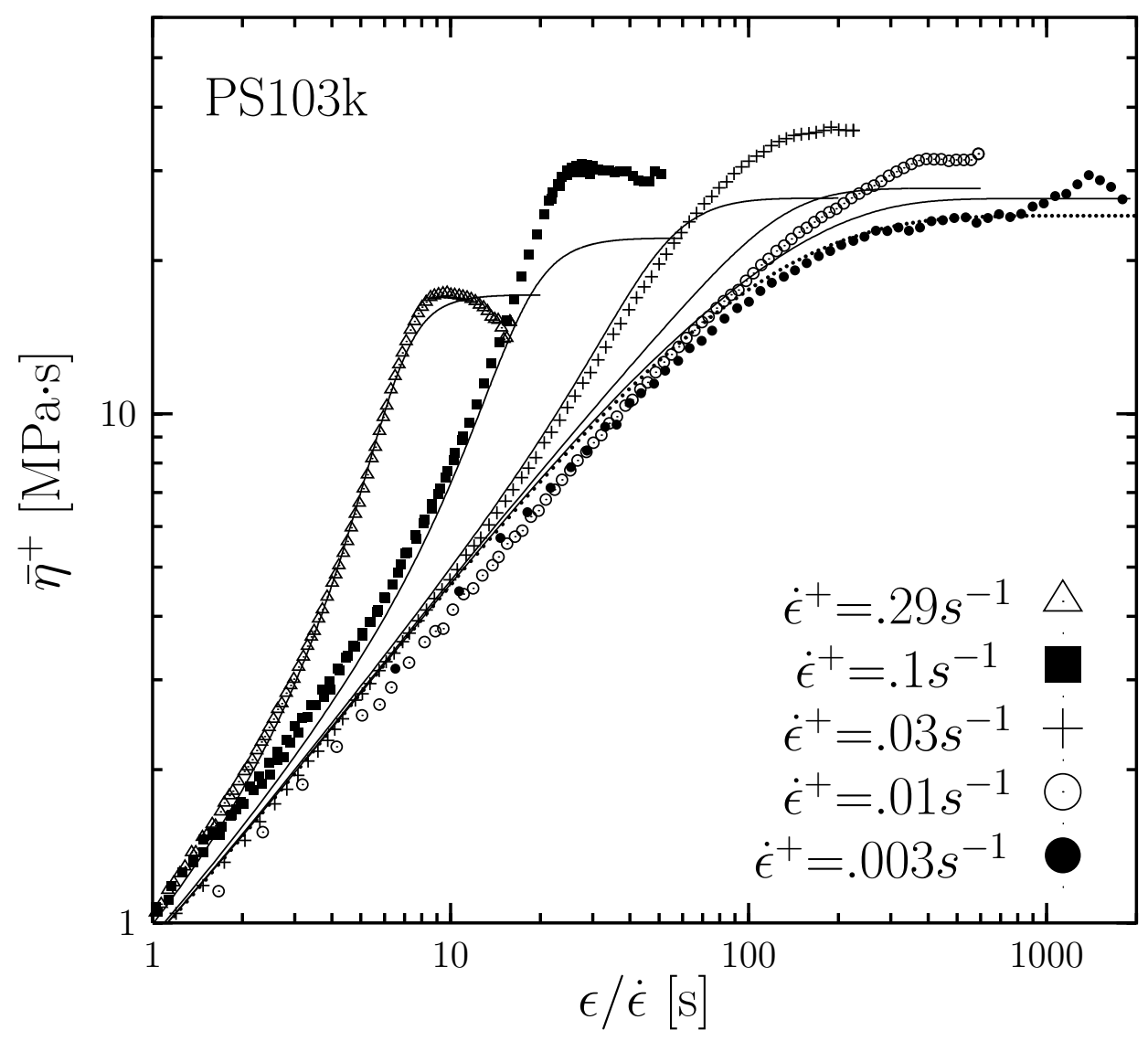

(b) 


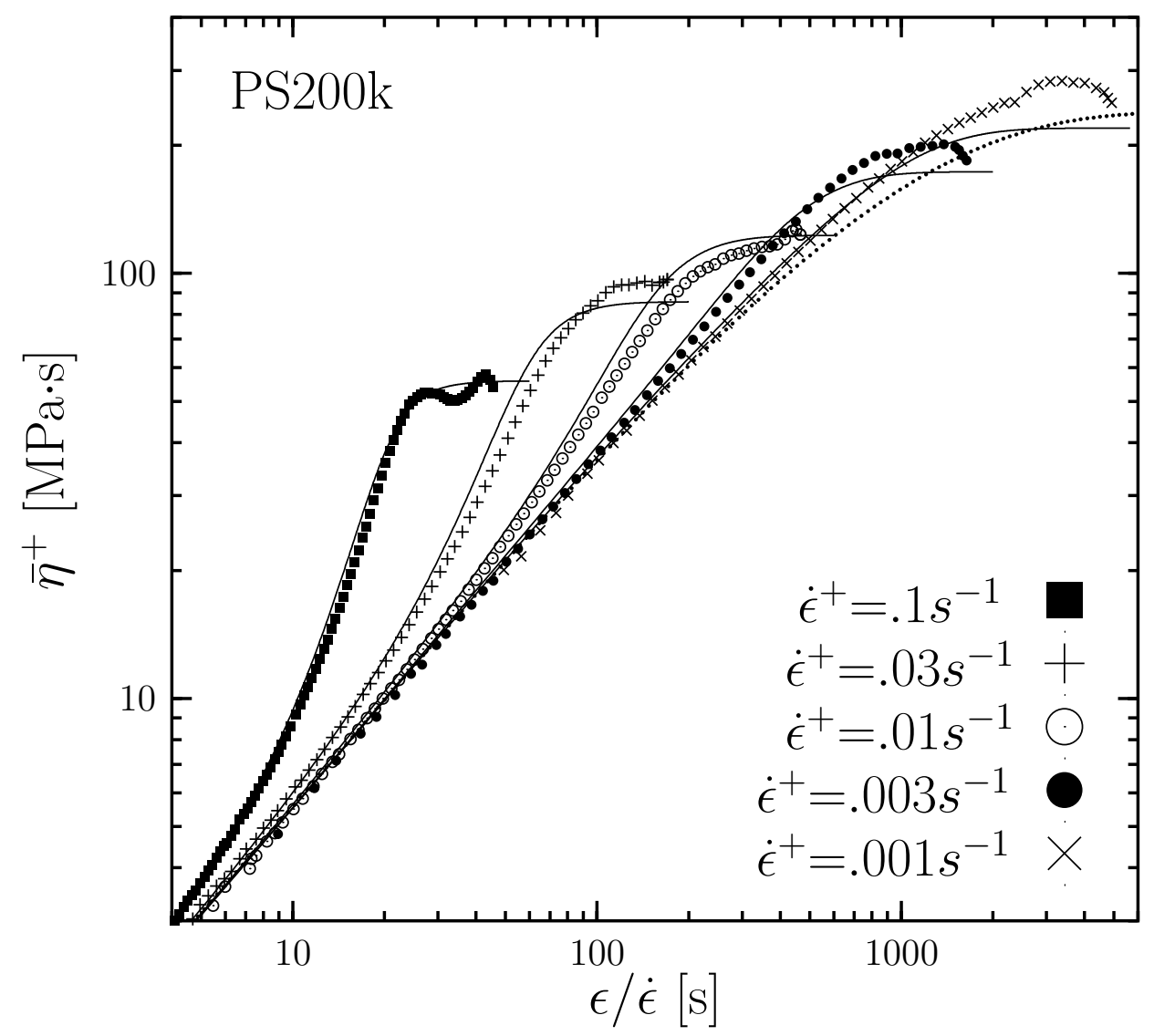

(c)

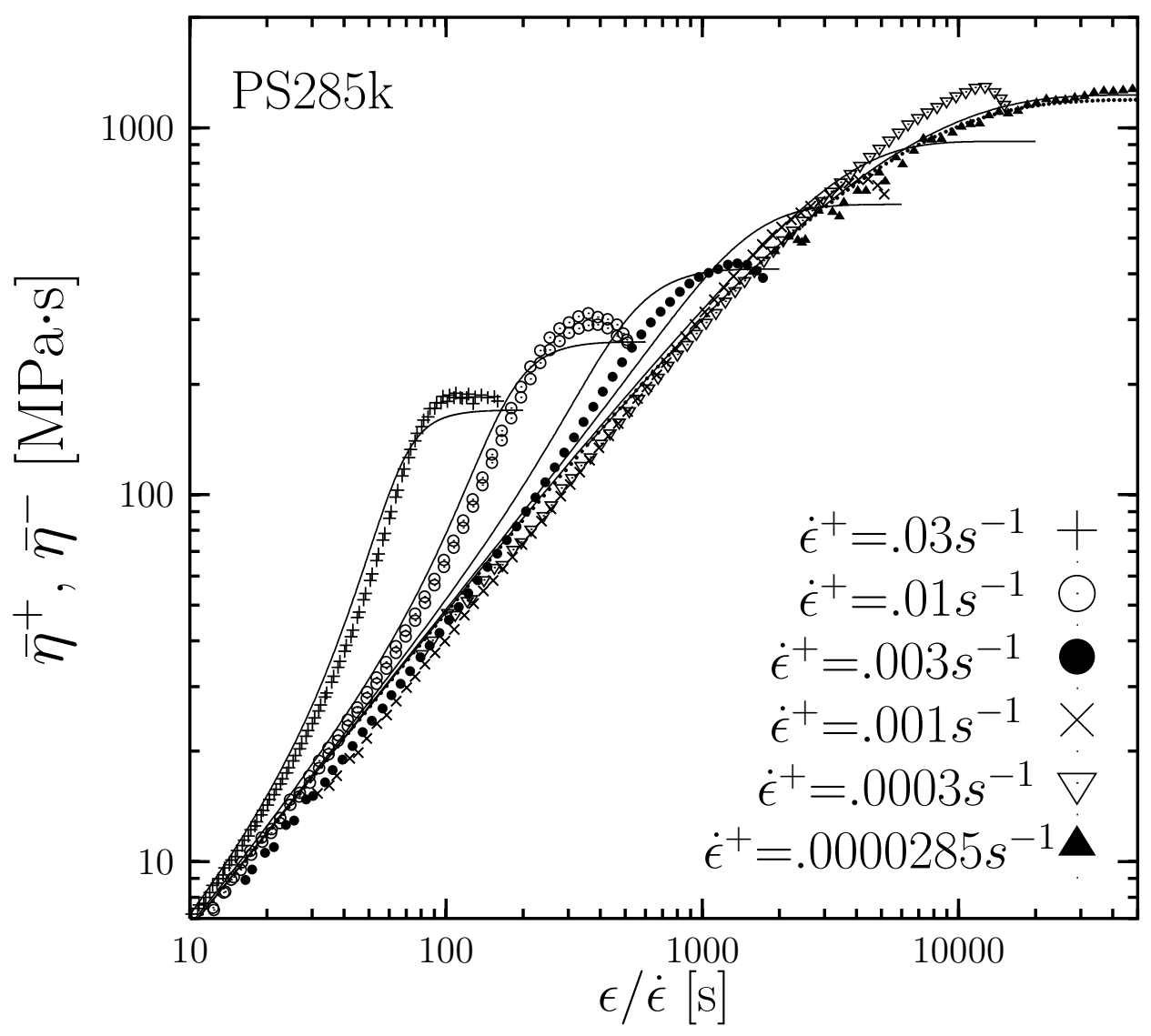

(d) 


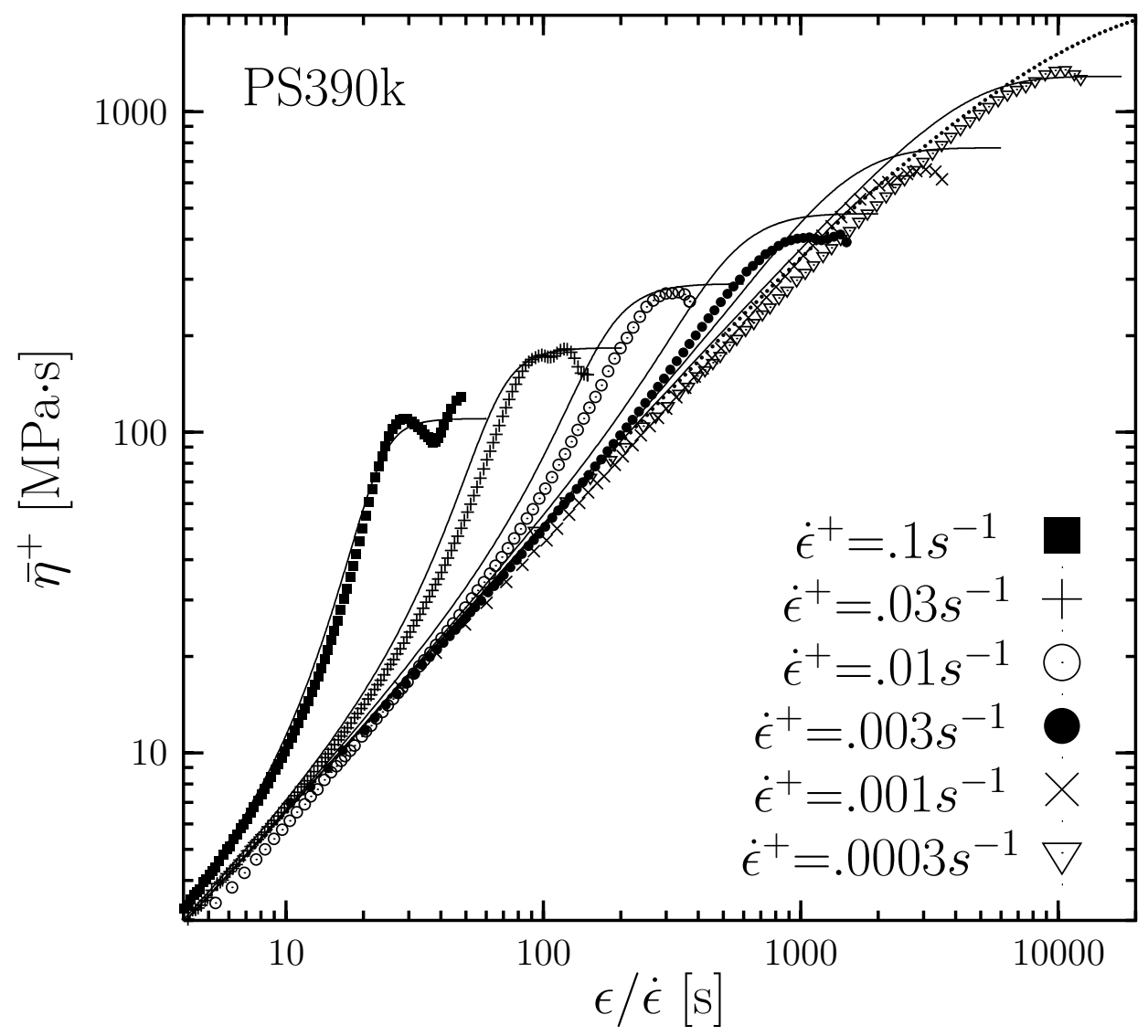

(e)

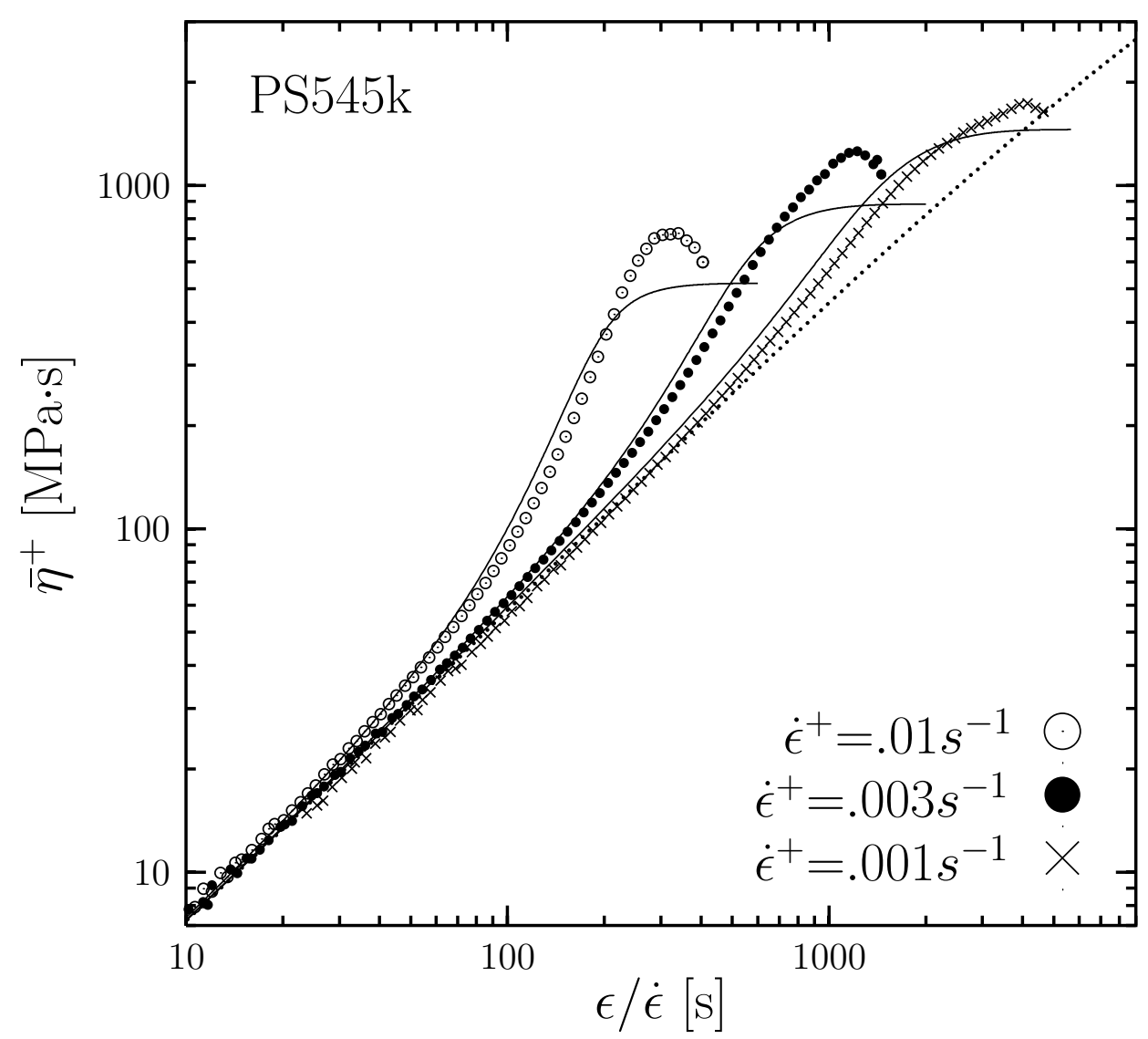

Figure 2: 


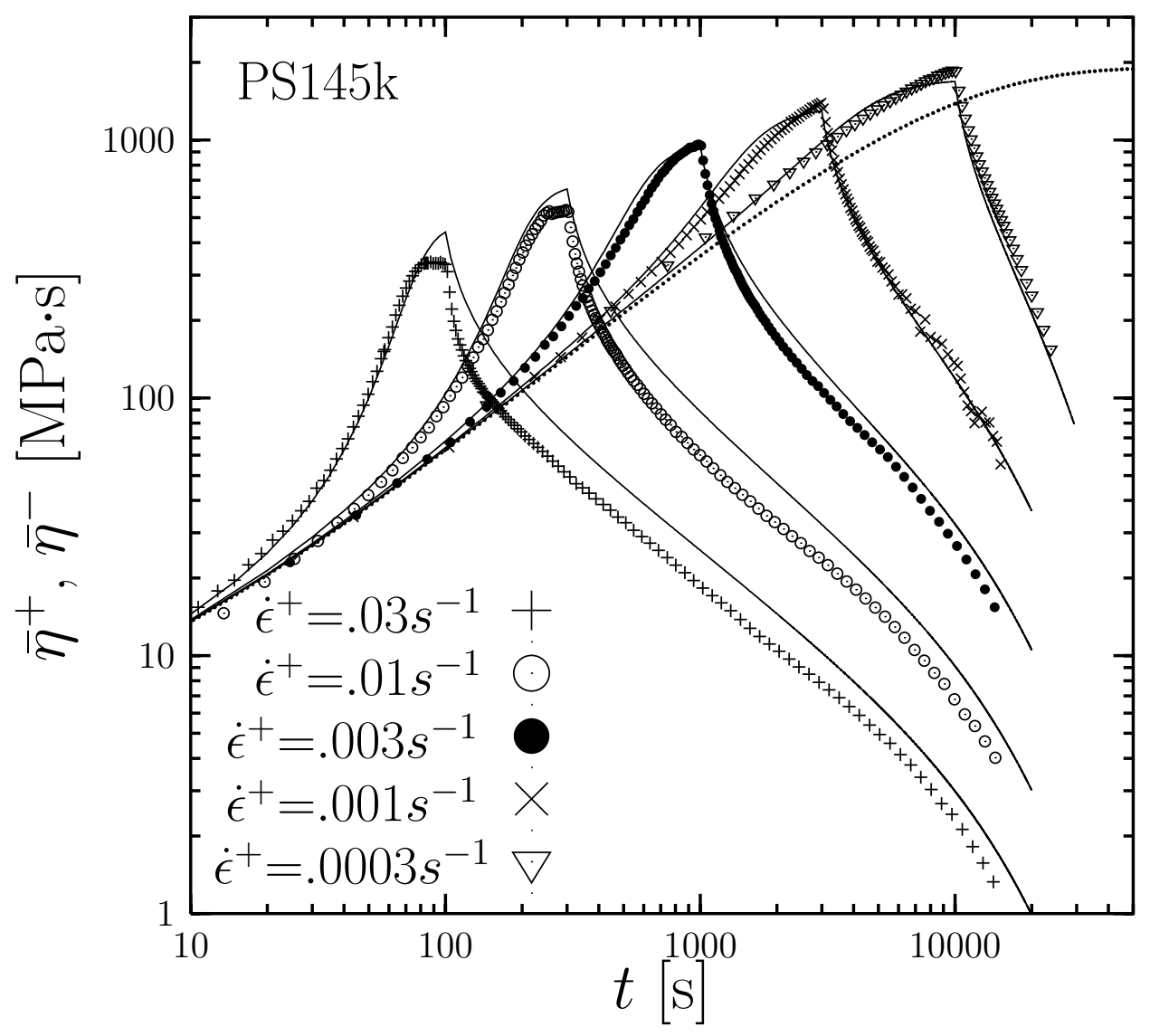

Figure 3: 


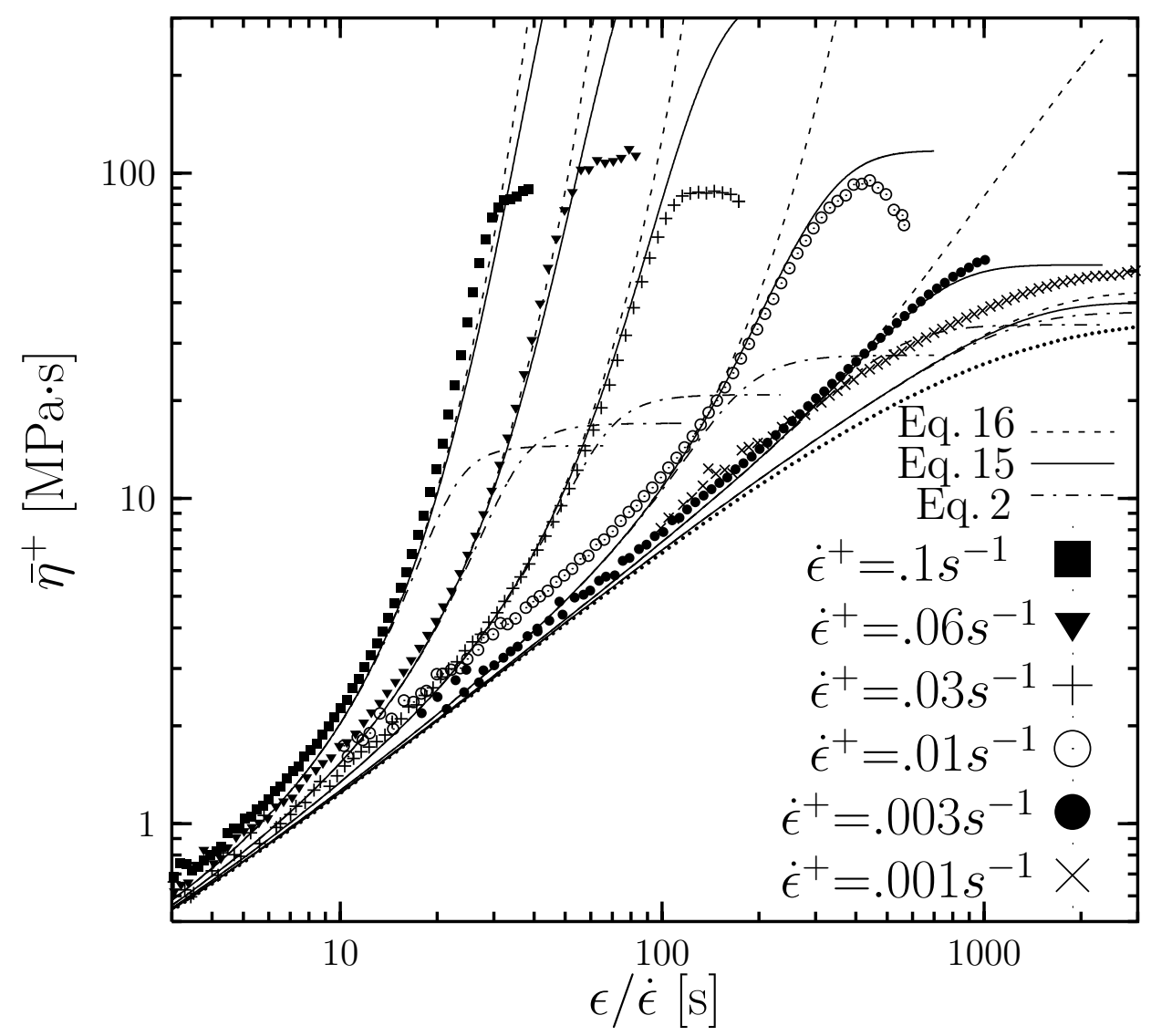

Figure 4: 


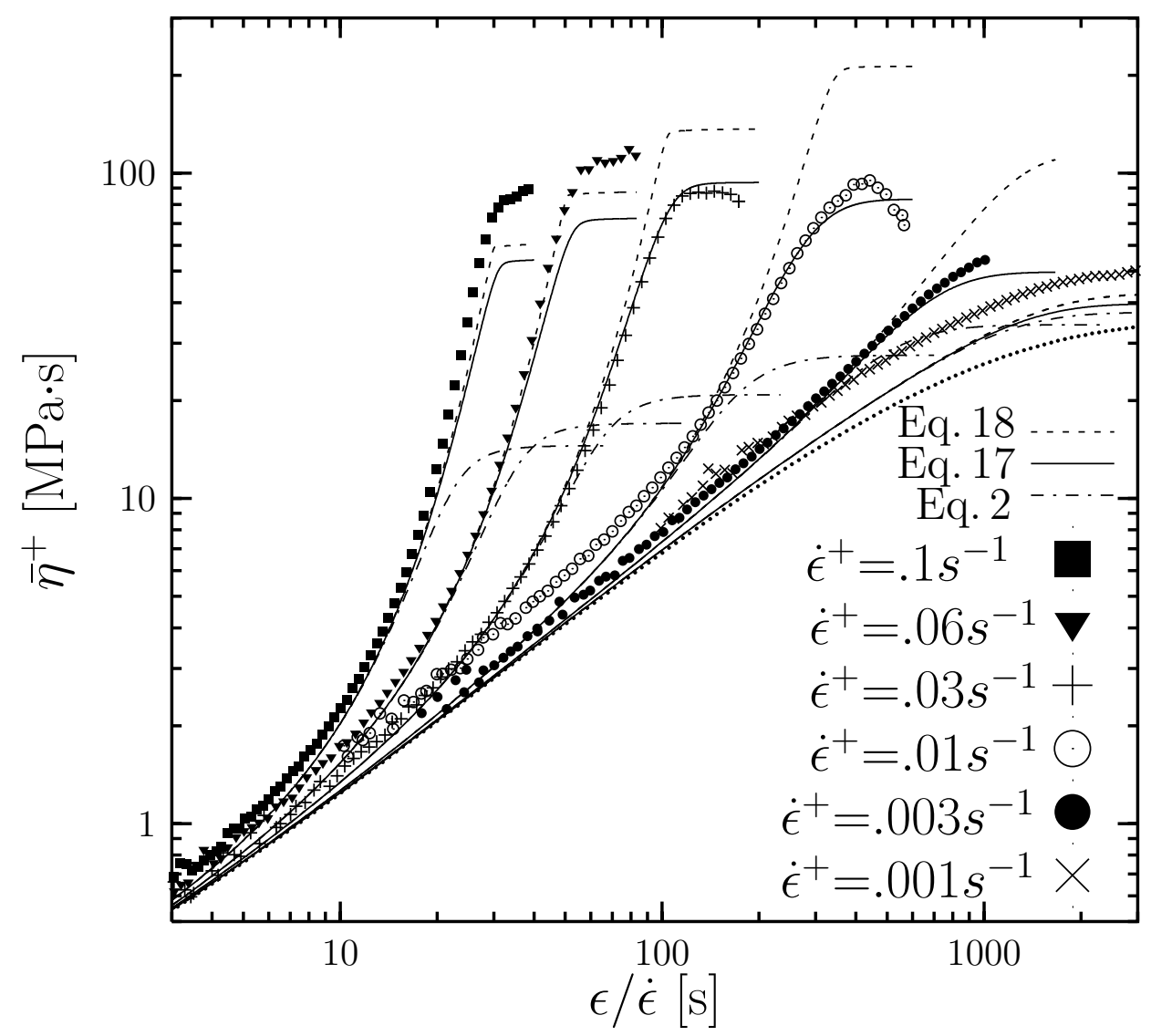

Figure 5: 


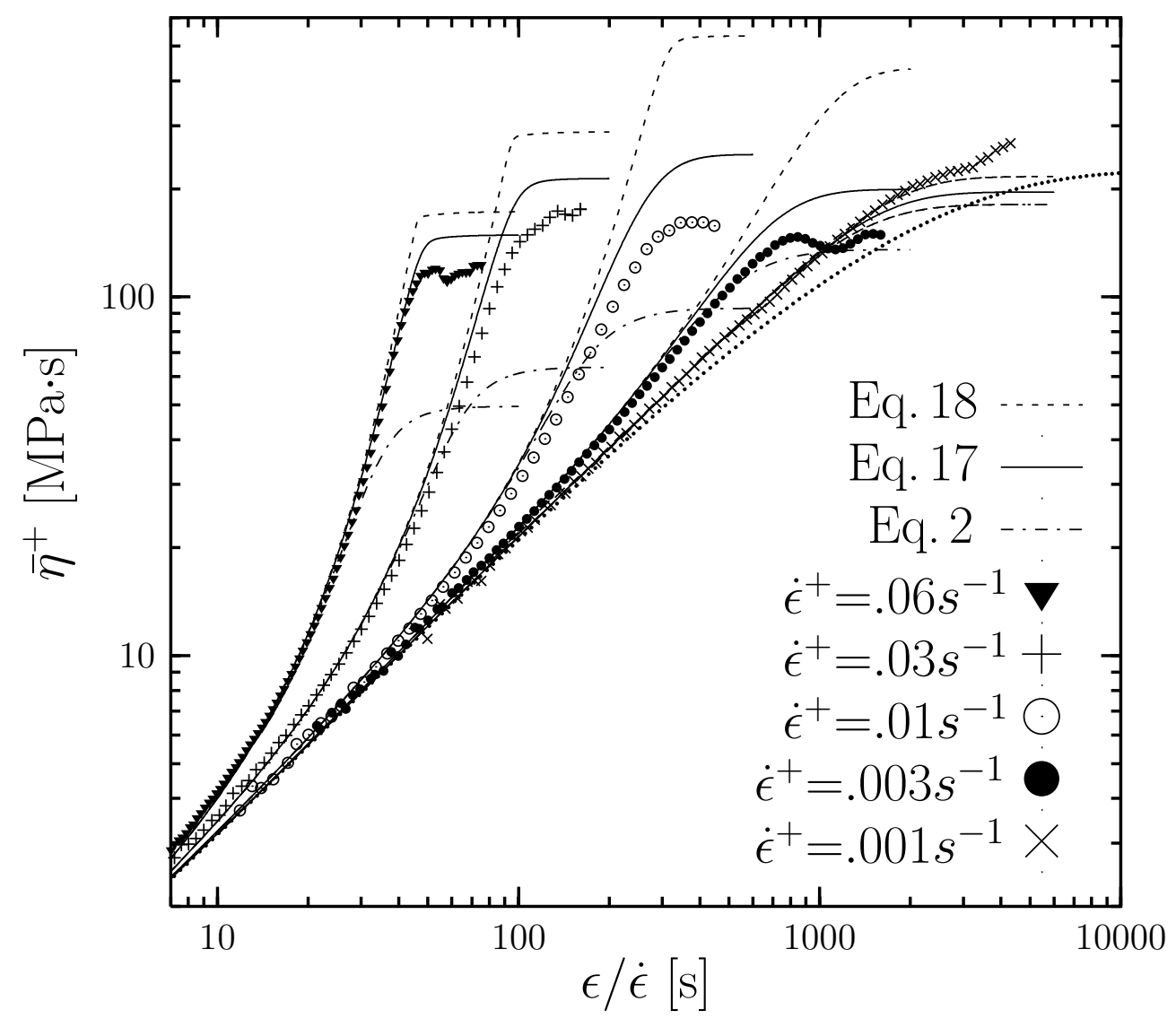

Figure 6: 


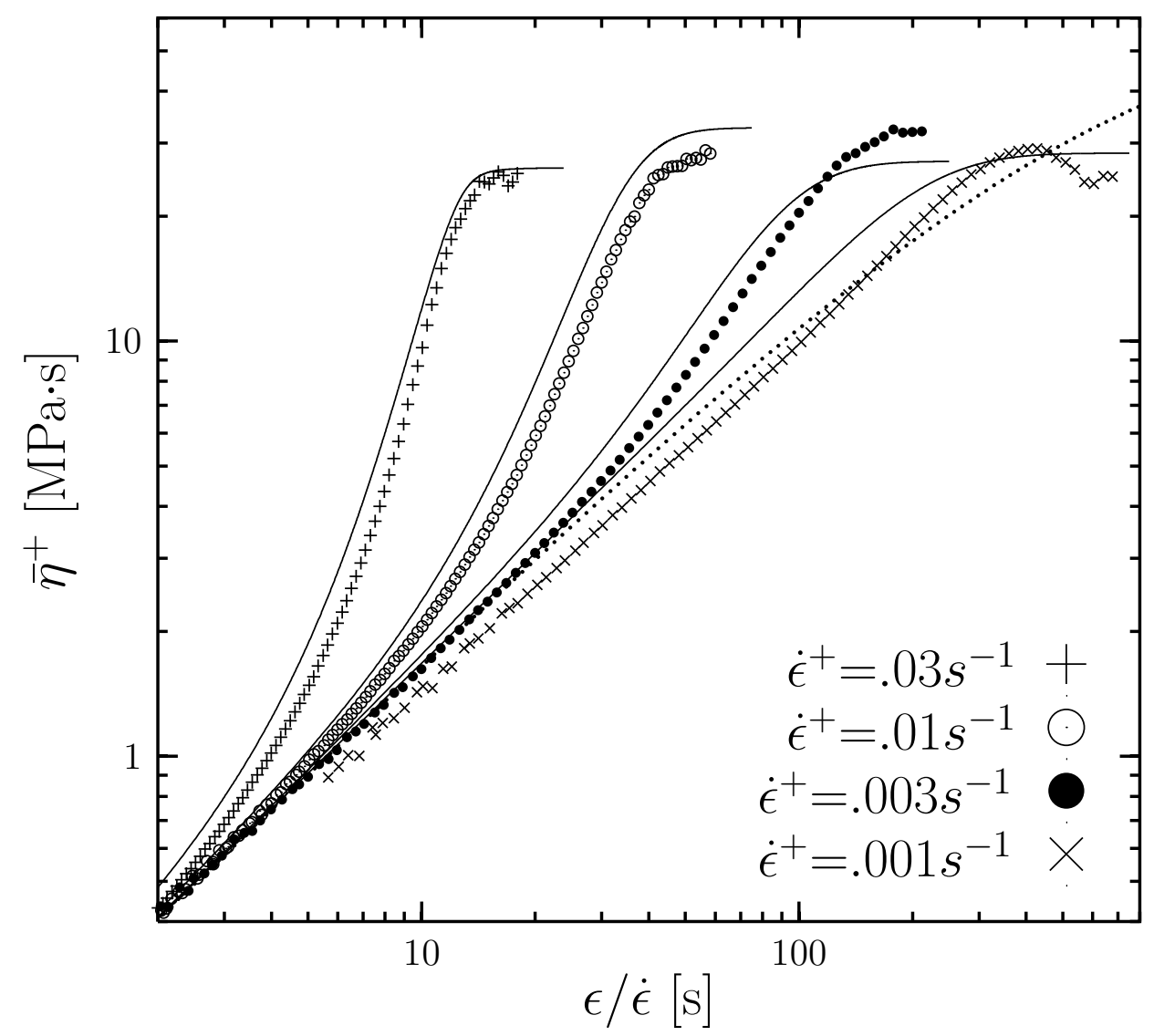

Figure 7: 


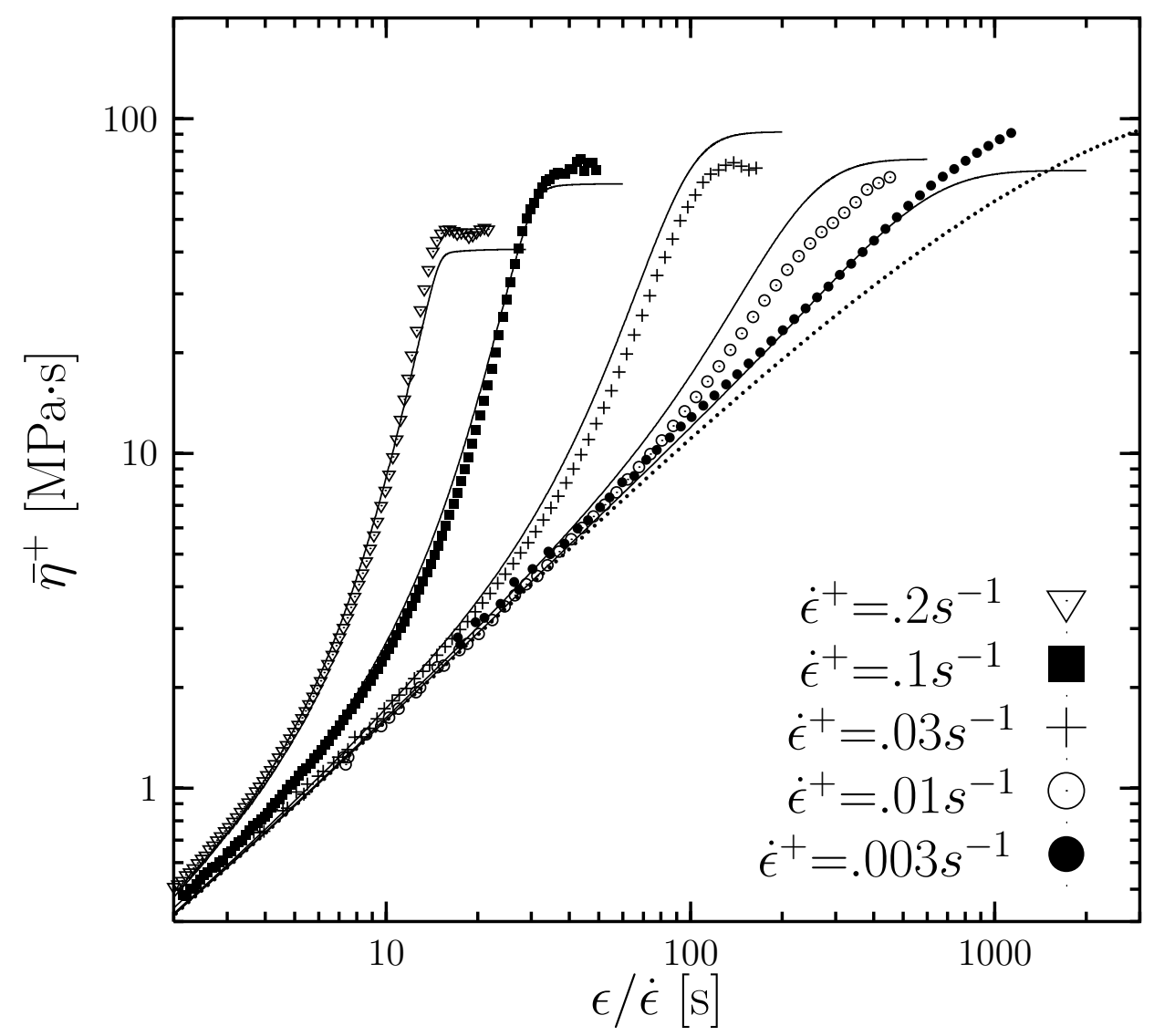

Figure 8: 


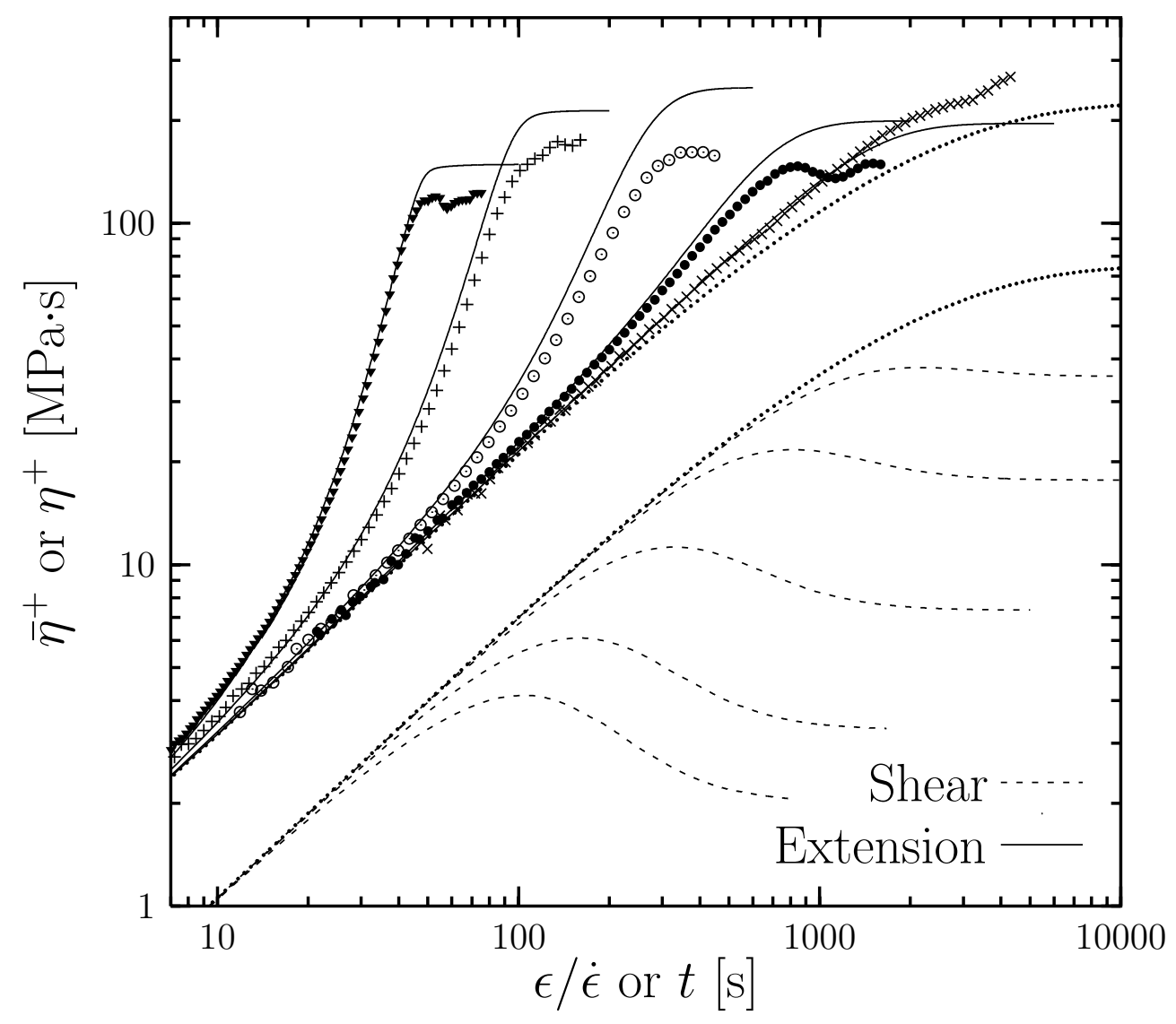

Figure 9: 


\section{References}

[1] Andreev M, Khaliullin RN, Steenbakkers RJA, Schieber JD (2013) Approximations of the discrete slip-link model and their effect on nonlinear rheology predictions. Journal of Rheology $57(2): 535-557$.

[2] Auhl D, Ramirez J, Likhtman AE, Chambon P, Fernyhough C (2008) Linear and nonlinear shear flow behavior of monodisperse polyisoprene melts with a large range of molecular weights. J Rheol 52(3):801-835.

[3] Bach A, Almdal K, Rasmussen HK, Hassager O (2003b) Elongational viscosity of narrow molar mass distribution polystyrene. Macromolecules 36(14):5174-5179.

[4] Bach A, Rasmussen HK, Hassager O (2003a) Extensional viscosity for polymer melts measured in the filament stretching rheometer. Journal of Rheology 47(2):429-441.

[5] Baumgaertel M, Winter HH (1992) Interrelation between continuous and discrete relaxation time spectra, Journal of Non-Newtonian Fluid Mechanics 44(1):15-36.

[6] Baumgaertel M, Schausberger A, Winter HH (1990) The relaxation of polymers with linear flexible chains of uniform length. Rheologica Acta 29(5):400-408.

[7] Bhattacharjee PK, Oberhauser JP, McKinley GH, Leal LG, Sridhar T (2002) Extensional rheometry of entangled solutions. Macromolecules 35(27):10131-10148.

[8] Boukany PE, Wang SQ, Wang XR (2009) Universal scaling behavior in startup shear of entangled linear melts. J Rheol 53(3):617-629.

[9] Cohen A (1991) A Padé approximant to the inverse Langevin function. Rheologica Acta $30(3): 270-273$.

[10] Currie PK (1982) Constitutive equations for polymer melts predicted by the Doi-Edwards and Curtiss-Bird kinetic theory models. Journal of Non-Newtonian Fluid Mechanics 11(1-2):53-68.

[11] De Gennes PG (1971) Reptation of a Polymer Chain in the Presence of Fixed Obstacles. The Journal of Chemical Physics 55(2):572571.

[12] Dhole S, Leygue A, Bailly C, Keunings R (2009) A single segment differential tube model with interchain tube pressure effect. Journal of Non-Newtonian Fluid Mechanics 161(1-3):10-18.

[13] Doi M, Edwards SF (1978) Dynamics of concentrated polymer systems. III. Constitutive equation. Journal of the Chemical Society, Faraday Transactions II 74(1):1818-1832.

[14] Doi M, Edwards SF (1986) The Theory of Polymer Dynamics; Clarendon Press: Oxford.

[15] Fang J, Kröger M, Öttinger HC (2000) A thermodynamically admissible reptation model for fast flows of entangled polymers. II. Model predictions for shear and extensional flows. Journal of Rheology 44(6):12931317.

[16] Giesekus H (1962) Elasto-viskose Flüssigkeiten, für die in stationären Schichtrömungen sämtliche Normalspannungskomponenten verschieden gross sind. Rheologica Acta 2(1):50-62.

[17] Huang Q, Alvarez NJ, Matsumiya Y, Rasmussen HK, Watanabe H, Hassager O (2013b) Extensional Rheology of Entangled Polystyrene Solutions Suggests Importance of Nematic Interactions. ACS Macro Letters 2(8):741-744. 
[18] Huang Q, Mednova O, Rasmussen HK, Alvarez NJ, Skov AL, Almdal K, Hassager O (2013a) Concentrated Polymer Solutions are Different from Melts: Role of Entanglement Molecular Weight. Macromolecules 46(12):5026-5035.

[19] Kolte MI, Rasmussen HK, Hassager O (1997) Transient filament stretching rheometer II: Numerical simulation. Rheologica Acta 36(3):285-302.

[20] Larson RG, Sridhar T, Leal LG, McKinley GH, Likhtman AE, McLeish TCB (2003) Definitions of entanglement spacing and time constants in the tube model. Journal of Rheology 47(3):809818.

[21] Likhtman AE, McLeish TCB (2002) Quantitative Theory for Linear Dynamics of Linear Entangled Polymers, Macromolecules 35(16):6332-6343.

[22] Marrucci G (1996) Dynamics of entanglements: A nonlinear model consistent with the Cox-Merz rule. Journal of Non-Newtonian Fluid Mechanics 62(2-3):279-289.

[23] Marrucci G, Ianniruberto G (2004) Interchain pressure effect in extensional flows of entangled polymer melts. Macromolecules 37(10):3934-3942.

[24] Marrucci G, Grizzuti N (1988) Fast flows of concentrated polymers - predictions of the tube model on chain stretching. Gazzetta Chimica Italiana 118(3):179-185.

[25] Menezes EV, Graessley WW (1982) Nonlinear rheological behavior of polymer systems for several shear-flow histories. Journal of Polymer Science part B - Polymer Physics 20(10):1817-1833.

[26] Milner ST, McLeish, TCB (1998) Reptation and contour-length fluctuations in melts of linear polymers. Physical Review Letters 81(3):725-728.

[27] Nielsen JK, Rasmussen HK, Hassager O (2008) Stress relaxation of narrow molar mass distribution polystyrene following uni-axial extension. Journal of Rheology 52(4):885-899.

[28] Nielsen JK, Rasmussen HK, Hassager O, McKinley GH (2006) Elongational viscosity of monodisperse and bidisperse polystyrene melts. Journal of Rheology 50(4):453-476.

[29] Osaki K, Inoue T, Isomura T, (2000) Stress Overshoot of Polymer Solutions at High Rates of Shear. Journal of Polymer Science part B - Polymer Physics 38(14):1917-1925.

[30] Osaki K, Inoue T, Uematsu T, Yamashita Y (2001) Evaluation methods of the longest Rouse relaxation time of an entangled polymer in a semidilute solution. Journal of Polymer Science part B - Polymer Physics 39(14):1704-1712.

[31] Park J, Mead DW, Denn MM (2012) Stochastic simulation of entangled polymeric liquids in fast flows: Microstructure modification. Journal of Rheology 56(5):1057-1081.

[32] Pearson DS, Kiss AD, Fetters LJ, Doi M (1989) Flow Induced Birefringence of Concentrated Polyisoprene Solutions. Journal of Rheology 33(3):517-535.

[33] Pearson DS, Kiss AD, Fetters LJ, Doi M (1990) Erratum: Flow Induced Birefringence of Concentrated Polyisoprene Solutions [J. Rheol. 33, 517 (1989)]. Journal of Rheology 34(4):613-613.

[34] Phan-Thien N., Tanner RI (1977) A new constitutive equation derived from network theory. Journal of Non-Newtonian Fluid Mechanics 2(4):353-365. 
[35] Rasmussen HK, Bejenariu AG, Hassager O, Auhl D (2010) Experimental evaluation of the pure configurational stress assumption in the flow dynamics of entangled polymer melts. Journal of Rheology 54(6):1325-1336.

[36] Rasmussen HK, Christensen JH, Gøttsche SJ (2000) Inflation of polymer melts into elliptic and circular cylinders. Journal of Non-Newtonian Fluid Mechanics 93(2-3):245-263.

[37] Rasmussen HK, Huang Q (2014) The missing link between the extensional dynamics of polymer melts and solutions. Journal of Non-Newtonian Fluid Mechanics dx.doi.org/10.1016/j.jnnfm.2013.11.002

[38] Schweizer T, Meerveld J, Öettinger HC (2004) Nonlinear shear rheology of polystyrene melt with narrow molecular weight distributionexperiment and theory. J Rheol 48(6):1345-1363.

[39] Spiegelberg SH, Ables DC, McKinley GH (1996) The role of end-effects on measurements of extensional viscosity in filament stretching rheometers. Journal of Non-Newtonian Fluid Mechanics 64(2-3):229-267.

[40] Szabo P (1997) Transient filament stretching rheometer part I: Force Balance analysis. Rheologica Acta 36(3):277-284 .

[41] Urakawa O, Takahashi M, Masuda T, Golshan Ebrahimi N (1995) Damping functions and chain relaxation in uniaxial and biaxial extensions: Comparison with the Doi-Edwards theory. Macromolecules 28(21):7196-7201.

[42] van Ruymbeke E, Nielsen J, Hassager O (2010) Linear and nonlinear viscoelastic properties of bidisperse linear polymers: Mixing law and tube pressure effect. Journal of Rheology 54(5):11551172 .

[43] Wagner MH, Kheirandish S, Hassager O (2005). Quantitative prediction of transient and steadystate elongational viscosity of nearly monodisperse polystyrene melts. Journal of Rheology 49(6):1317-1327.

[44] Wagner MH, Rolón-Garrido VH (2010) The interchain pressure effect in shear rheology. Rheologica Acta 49(5):459-471.

[45] Yaoita T, Isaki T, Masubuchi Y, Watanabe H, Ianniruberto G, Marrucci G (2011) Primitive Chain Network Simulation of Elongational Flows of Entangled Linear Chains: Role of Finite Chain Extensibility. Macromolecules 44(24):9675-9682.

[46] Yaoita T, Isaki T, Masubuchi Y, Watanabe H, Ianniruberto G, Marrucci G (2012) Primitive Chain Network Simulation of Elongational Flows of Entangled Linear Chains: Stretch/Orientation-induced Reduction of Monomeric Friction. Macromolecules 45(6):27732782 .

[47] Ye X, Sridhar T (2005) Effects of the polydispersity on rheological properties of entangled polystyrene solutions, Macromolecules 38(8):3442-3449. 